\title{
Designing and Psychometric Evaluation of Morbid Obesity Quality of Life Questionnaire: A Mixed Methods Study
}

\author{
Negar Yazdani ${ }^{1,2}$, Farkhondeh Sharif ${ }^{3,},{ }^{*}$ Nasrin Elahi ${ }^{1}$, Abbas Ebadi $^{4}$ and Vahid Hosseini ${ }^{5}$ \\ ${ }^{1}$ Nursing Care Research Center in Chronic Diseases, Ahvaz Jundishapur University of Medical Sciences, Ahvaz, Iran \\ 2 Neonatal Research Center, Shiraz University of Medical Sciences, Shiraz, Iran \\ ${ }^{3}$ Community-based Psychiatric Care Research Center, Shiraz University of Medical Sciences, Shiraz, Iran \\ ${ }^{4}$ Behavioral Sciences Research Center, Lifestyle Institute, Faculty of Nursing, Baqiyatallah University of Medical Sciences, Tehran, Iran \\ 5 Colorectal Research Center, Shiraz University of Medical Sciences, Shiraz, Iran
}

* Corresponding author: Farkhondeh Sharif, Community-based Psychiatric Care Research Center, Shiraz University of Medical Sciences, Shiraz, Iran. Email: fsharif2008@gmail.com

Received 2020 September 28; Revised 2020 0ctober 19; Accepted 2020 October 25.

\begin{abstract}
Background: Morbid obesity negatively affects all aspects of quality of life (QOL); therefore, the assessment of QOL can be a useful criterion to evaluate obesity outcome.

Objectives: The present study aimed to design a QOL questionnaire in morbid obesity and assess its validation features.

Methods: This sequential-exploratory mixed-methods study was conducted in both qualitative and quantitative stages. The qualitative stage was carried out through 20 semi-structured interviews with morbidly obese people. Furthermore, the initial items of the questionnaire were obtained by a review of related literature. In the next stage, face, content, and construct validity were assessed. The construct validity was examined by exploratory factor analysis with 319 people. Moreover, Cronbach's alpha and test-retest correlation coefficient were calculated to evaluate the reliability of the questionnaire. Furthermore, confirmatory factor analysis was assessed.

Results: Based on the results of the quantitative stage, an 80 -item pool was extracted. The exploratory factor analysis yielded six factors that explained $50.62 \%$ of the variance. The factors of one to six were as follows: self-dissatisfaction (15 items), functional disorders (7 items), spiro-mental experiences (11 items), perso-social isolation ( 7 items), physical changes ( 7 items), and financial pressure (3 items). The final Cronbach's alpha coefficient of the 50 -item questionnaire was calculated at 0.94 . Moreover, the intra-class correlation coefficient between the test and retest scores was estimated at 0.97. All indicators of confirmatory factor analysis demonstrated that goodness of fit was acceptable for each factor. Furthermore, this questionnaire had appropriate interpretability, feasibility, and did not have any floor and ceiling effects.

Conclusion: This valid and reliable questionnaire can be used as a suitable measurement tool for assessing the QOL of morbidly obese people in Iran and other countries with similar cultural features. This questionnaire can improve the quality of future clinical research on obesity. In addition, health care providers can perform a peculiar role in the provision of efficient services to patients to prevent obesity and its associated complications.
\end{abstract}

Keywords: Morbid obesity, Psychometrics, Quality of life, Questionnaire, Reliability, Validity

\section{Background}

Morbid obesity (MO), as a multifactorial and public health problem (1), has increased across the globe due to lifestyle changes (2). Based on global statistics, more than 1.9 billion and 600 million people are overweight and obese, respectively (3). An Iranian systematic review reported that $25.2 \%$ of women and $15 \%$ of men had body mass index (BMI) $>30$ (4).

Although MO causes dangerous physical, mental, and socio-economic problems, and even poor quality of life (QOL) (5), it is a preventable and curable disease (6). Different strategies have been developed for the management of obesity, including a combination of nutritional, physical activity, cognitive-behavioral, and surgical approaches $(7,8)$. Some studies have addressed the major barriers to obesity management, such as the lack of needed instruments, training, and time. Novel treatment methods help clinical professionals to make these interventions and support weight management in the primary care setting (9).

Some generic tools, such as Short Form-36 (SF36 ), can be used for the evaluation of QOL (10); nonetheless, these tools cannot measure all specific aspects of morbid obesity-related problems. In this regard, a comparative study has pointed to differences in types of QOL measurement tools (e.g., specific and generic tool) and highlighted the importance of using both general and specific tools to conduct valid studies (11).

On the other hand, there are specific instruments to measure QOL and obesity-related problems; however, they have been designed in different cultures and religions. Moreover, they suffer from some weaknesses, which have been mentioned in another article separately (12). For instance, previously conducted studies demonstrated that some tools have measured QOL only for patients who had undergone surgery. Moreover, some dimensions of QOL (e.g., spirituality) and all types of psychometric properties have not been addressed in previous studies. They have also used a deductive

Copyright (C) 2020, Author(s). This is an open-access article distributed under the terms of the Creative Commons Attribution-NonCommercial 4.0 International License (http://creativecommons.org/licenses/by-nc/4.0/) which permits copy and redistribute the material just in noncommercial usages, provided the original work is properly cited 
approach to design tools, which had not been based on lived experiences of morbidly obese people (1315). Since life with obesity and its QOL are two context-based phenomena, reflecting on obesity and measurement of its effects on health in the context of life can provide deeper insight into obesity-related problems. Moreover, they may help advance a more comprehensive approach into new dimensions of QOL (e.g., religion and spirituality) in obesity health care (16-18).

On the other hand, due to different effects of sociocultural context on the concepts, different patterns of obesity, nutrition, QOL concept in every culture (19), the prevalence of obesity, and lack of specific and context-based QOL instrument in our health system, it seems necessary to design a valid, reliable, and experience-based instrument in our context (20).

\section{Objectives}

The current study aimed to design a morbid obesity QOL questionnaire (MOQOLQ) and to assess its validation features.

\section{Methods}

\subsection{Research Design}

This sequential-exploratory mixed-methods study was conducted in both qualitative and quantitative phases. The qualitative stage involved generating items and designing an instrument, while the quantitative stage entailed reducing the items and assessing the validation features of the questionnaire.

\subsubsection{First stage: Item generation}

In this stage, semi-structured interviews were performed with 20 morbidly obese patients based on conventional content analysis. This phase was carried out in obesity and nutrition clinics affiliated to Shiraz University of Medical Sciences within May 2016January 2017 in Shiraz, which is a referring center for MO in the south of Iran. The patients, who had referred from different regions, were assessed by an eight-member health care team (e.g., clinicians, nurses, nutrition experts, sports experts, and psychologists) in these clinics.

The inclusion criteria entailed the patients $>18$ years old with body mass index (BMI) $\geq 40 \mathrm{Kg} / \mathrm{m}^{2}$ or $\mathrm{BMI}>35 \mathrm{Kg} / \mathrm{m}^{2}$ with co-morbidity. On the other hand, the patients who suffered from serious physical or mental illnesses or were not willing to take part in the study were excluded. It is noteworthy that all of the participants spoke Persian and were from different regions. To this aim, the purposeful sampling method was used and continued until data saturation (21). Although data saturation was achieved, and no new code was derived in the $15^{\text {th }}$ interview, five more interviews were conducted to assure that enough data were collected. Data were analyzed using Graneheim-Lundman (22).

The item pool was retrieved from the results of this phase (an inductive approach) and the literature review (a deductive approach) (23). Eventually, the research team, including a surgeon, tool designer, statistics expert, and faculty members of nursing school, revised the initial tool for the validation stage.

\subsubsection{Second stage: Reduction of items and assessment of validation features}

Throughout the second stage, participants were selected via the convenience sampling method and based on previous inclusion and exclusion criteria that were mentioned in the first stage.

\subsubsection{Face Validity}

Both quantitative and qualitative face validity was assessed in this stage. The face validity was qualitatively evaluated during direct interviews with MO people $(n=10)$. In this regard, the participants read the items out loud. They were requested to describe the concept of items and identify the unsuitable or ambiguous expressions so that the items could be revised (24). Moreover, two literature experts were asked to score the clarity, simplicity, and comprehensibility of each item. As a result, some items were modified and corrected according to patients' opinions. Subsequently, the importance of each item was determined by quantitative face validity.

In this regard, another group of MO patients $(n=10)$ were asked to score each item in terms of its importance by a five-point Likert scale (1= not important to 5=very important). Thereafter, the impact score of each item was assessed using the following formula:

Impact score of item=frequency $(\%) \times$ importance

Finally, the items lower than 1.5 were removed (25).

\subsubsection{Content Validity}

The content validity of the instrument was assessed using two approaches (i.e., quantitative and qualitative). A panel of 10 experts was formed, including three surgeons, one nutritionist expert, three nursing instructors, two psychologists, and one instrument designer specialist. They were faculty members of different universities in Iran with the work experience of $\geq 3$ and clinical work experience. To calculate the content validity rate (CVR), the panel was requested to give a score of 1-3 to each item ( $3=$ essential, 2= useful but not essential, and $1=$ not essential) (26).

$$
C V R=\frac{n E-\frac{N}{2}}{\frac{N}{2}}
$$

According to the table of Lawshe, 0.62 is the 
minimum standard level to accept the content validity ratio for 10 specialists (27).

To evaluate the content validity index (CVI), 10 specialists were requested to rate each item based on a four-point Likert scale (from 1=unrelated to $4=$ completely related). Thereafter, CVI was calculated using the following formula:

\section{$\mathrm{CVI} \equiv \frac{\text { number of raters giving a rating of ' } 3 \text { ' or ' } 4 \text { ' }}{\text { total number of raters }}$}

Subsequently, the adjusted Cohen's kappa coefficient was estimated. The adjusted Kappa coefficient of less than 0.74 was regarded as the standard to remove each item. Furthermore, a value of 0.9 was considered acceptable for the scale-level CVI (averaging the item-level CVIs). Finally, a pilot study was conducted on MO patients $(n=40)$ to investigate item analysis. To this end, Cronbach's alpha coefficient and loop method were investigated evaluating the inter-item and itemtotal correlation (28).

\subsubsection{Construct Validity}

In the present study, the construct validity was assessed using exploratory factor analysis (EFA), convergent and divergent, and known-group validity. Sampling was conducted using the convenience sampling method, and the sample size was calculated based on the following formula (29):

\section{$n=3$ to $10 \times$ the numbers of questions}

Therefore, the mean score of the sample size was used ( 6.5 times the number of questions) in our study $(6.5 \times 52$ questions $=338$ patients $)$. The population of the study were selected from two main hospitals of Shiraz and Ahwaz, Iran within July 2018-January 2019. Finally, 319 questionnaires were involved in the final analysis. Thereafter, the main assumptions of factor analysis were evaluated. In this regard, the adequacy of the sample size was assessed using the Kaiser-Meyer-Olkin (KMO) test.

Furthermore, the correlation matrix was evaluated using Bartlett's test. KMO values between 0.7 and 0.8 are indicative of moderately adequate sample size, and KMO values of $\geq 0.9$ reveal good adequacy of the sample size. The principal axis factoring and Varimax rotation were used to identify the extraction of latent factors $(30,31)$. The factor loading less than 0.40 was considered a standard value (25). To evaluate the convergent validity, the 12-Item Short-Form Health Survey (SF-12) questionnaire was used, which was validated in other studies $(32,33)$.

Moreover, the divergent validity was assessed by calculating the relationship between this tool and body image concern inventory (BICI), which was validated in previous studies $(34,35)$. After evaluating the convergent and divergent validity, we examined if groups with different BMI could be distinguished based on their QOL scores.

Hypothesis: Higher scores were expected in people who have $\mathrm{BMI}<40$, compared to those with $\mathrm{BMI} \geq 40$ (36).

\subsubsection{Reliability}

To determine the reliability of the questionnaire, internal consistency and stability were evaluated. Internal consistency was evaluated using Cronbach's alpha coefficient. A Cronbach's alpha coefficient of $>0.7$ indicates a reasonable internal consistency (37). Stability was evaluated using the test-retest technique. To this aim, 20 MO patients completed the questionnaire twice, with a test-retest interval of 1014 days. Thereafter, the intra-class correlation coefficient (ICC) test was used to compare the scores of the two stages. ICC values equal to and greater than 0.8 were regarded as acceptable levels of stability (38).

Furthermore, to verify the absolute reliability, the standard of measurement error (SEM) was calculated:

$$
S E M=\sqrt{(1-I C C)} \times S D
$$

In addition to consistency, the agreement was also measured. To confirm the positive agreement, the minimal important change (MIC) must be more than the smallest detectable change (SDC) (39). Furthermore, confirmatory factor analysis (CFA) was carried out on new samples who were selected by convenience sampling method, and the sample size was calculated based on the following formula $(q=$ the number of variables or factors) (40).

$$
5 q<n<20 q
$$

\subsubsection{Assessment of Interpretability and Floor and Ceiling Effects}

The interpretability was defined by the ability of tool for the identification of treatment response (37). Furthermore, the present study considered floor and ceiling effects which were considered present if $>15 \%$ of participants achieved the minimum score/floor score $(50 / 250)$ or maximum/ceiling score (250/250) score (41).

\subsubsection{Ethical Consideration}

The present study was approved by the Ethics Committee of Ahvaz University of Medical Sciences (IR.AJUMS.REC.2017, 10). Written informed consent was obtained from all participants, and they were informed of ethical, confidentiality (anonymity in publishing), and voluntary participation principles.

\subsubsection{Statistical Analysis}

The obtained data were analyzed in SPSS software (version 16) and Mplus software (version 6.1). Moreover, the mean, standard deviation, 
frequency, and percentage of frequency were calculated. In addition, EFA, variance analysis, Cronbach's alpha coefficient, internal consistency coefficient, independent T-test, paired T-test, and Pearson correlation were utilized to analyze the data. Weighted least squares mean and variance adjusted (WLSMV) estimation procedure was used to fit the categorical confirmatory factor analysis (CCFA). A p-value less than 0.05 was considered statistically significant.

\section{Results}

\subsection{First stage: Item generation}

After analyzing participants' interviews, we extracted 104 items based on participants' opinions and literature review. Finally, the revised pool included 80 items.

\subsection{Second stage: Selection of the Items and assessment of face validity}

Inappropriate and vague expressions were revised in the evaluation of face validity. Moreover, the impact score of all the achieved items was more than 1.5 (range=1.54-4.91).

\subsubsection{Content Validity}

To evaluate the qualitative content validity of the questionnaire, specialists edited the transcribed structure of some items, yielding 10 items. To this end, 18 items with a CVR of less than 0.62 were removed. In this phase, the 57 remaining items obtained a satisfactory adjusted Kappa coefficient. The CVI of the scale was calculated at 0.98, demonstrating the desirable quality of content validity. Subsequently, the item analysis was conducted on 40 patients, and five items, which had more correlation with other items, were combined. Finally, a 52- item questionnaire, which had two types of five-point Likert scales (ranging from very much to very little and from always to never), were used for the validation stage.

\subsubsection{Construct Validity}

The participants' demographic data are presented in Table 1. Their mean age, weight, height, and BMI were obtained as $38.90 \pm 9.90 \mathrm{Y} / 0,116.40 \pm 20.80 \mathrm{Kg}$, $163.70 \pm 8.8 \mathrm{~cm}$, and $43.20 \pm 5.40 \mathrm{Kg} / \mathrm{m}^{2}$, respectively.

In the current study, principal axis factoring and Varimax rotation were used for performing EFA. The KMO index was calculated at 0.90 signifying the adequate sample size for performing FA. Bartlett's test revealed a significant correlation among all items $(\chi 2=8448.84$, df: 1326 , and $\mathrm{P}=0.001)$ which is indicative of the suitability of this model for FA. Thereafter, Varimax rotation (Eigenvalues more than one) was used to extract suitable factors. The eigenvalues of the first to sixth hidden factors were estimated at 15.46, 3.28, 2.58, 1.93, 1.61, and 1.43,

\begin{tabular}{llc}
$\begin{array}{l}\text { Table 1. Demographic Characteristics of } \\
(\mathrm{n}=319)\end{array}$ & & \\
\hline Variable & Male & Participants \\
\hline Gender & Female & $64(20.10)$ \\
& Single & $225(79.90)$ \\
Marital status & Married & $65(20.40)$ \\
& Divorced & $239(74.90)$ \\
& Widowed & $3(0.90)$ \\
& < Diploma & $1(0.80)$ \\
Education level & Diploma & $75(23.50)$ \\
& Associate degree & $124(38.90)$ \\
& Bachelor degree & $30(9.40)$ \\
& Master degree & $67(21)$ \\
Job & Housewife & $23(7.20)$ \\
& Employee & $180(56.40)$ \\
& Business man & $74(23.20)$ \\
& Unemployed & $47(14.70)$ \\
City & Shiraz & $18(5.60)$ \\
& Fars province & $90(28.21)$ \\
Total & Ahvaz & $65(20.38)$ \\
\hline & Other cities & $77(24.14)$ \\
& & $87(27.27)$ \\
& & $319(100)$ \\
\hline
\end{tabular}

respectively. The eigenvalue of $>0.4$ was considered to keep an item. Meanwhile, the variables which had more correlation with others were classified in one subscale. As illustrated by the findings, two items were removed from the questionnaire due to poor factor loading. Based on the Scree plot, six factors were extracted to enable us to perform the construct validity of MOQOLQ (Figure 1). These factors covered $50.62 \%$ of the total variance. After rotation, the first to sixth factors explaining variances were calculated at $16 \%, 9.03 \%, 8.16 \%, 6.23 \%, 6.15 \%$, and $5.02 \%$, respectively. The first to sixth factors were named self-dissatisfaction (15 items), functional disorders (7 items), spiro-mental experiences (11 items), persosocial isolation (7 items), physical changes ( 7 items), and financial pressure ( 3 items). Table 2 presents the factor loading of each item (range $=0.40$ to 0.78 ). Furthermore, CFA was evaluated with 300 new MO patients from August 2019 to December 2019 in Shiraz, Iran.

All indicators achieved from CFA pointed to the acceptability of goodness of fit for each factor (Table 3). Moreover, Figure 2 displays the Path diagram of the CFA of the MOQOLQ. The mean values of the six dimensions of the MOQOLQ were as follows: selfdissatisfaction: $36.35 \pm 14.31$, functional disorders:

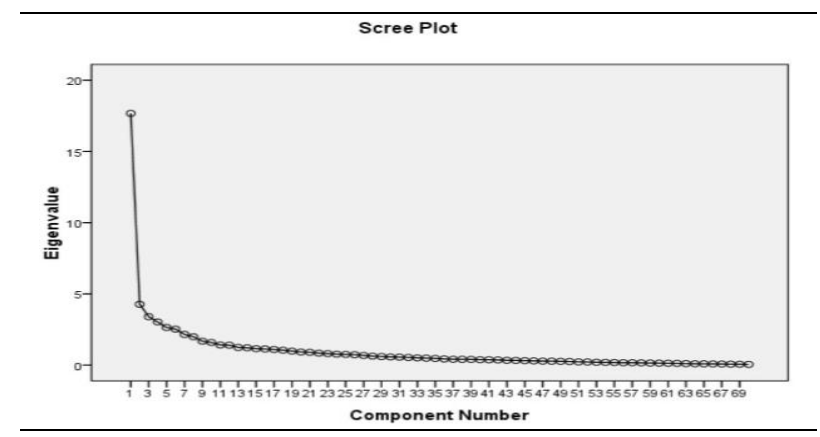

Figure 1. Scree plot 


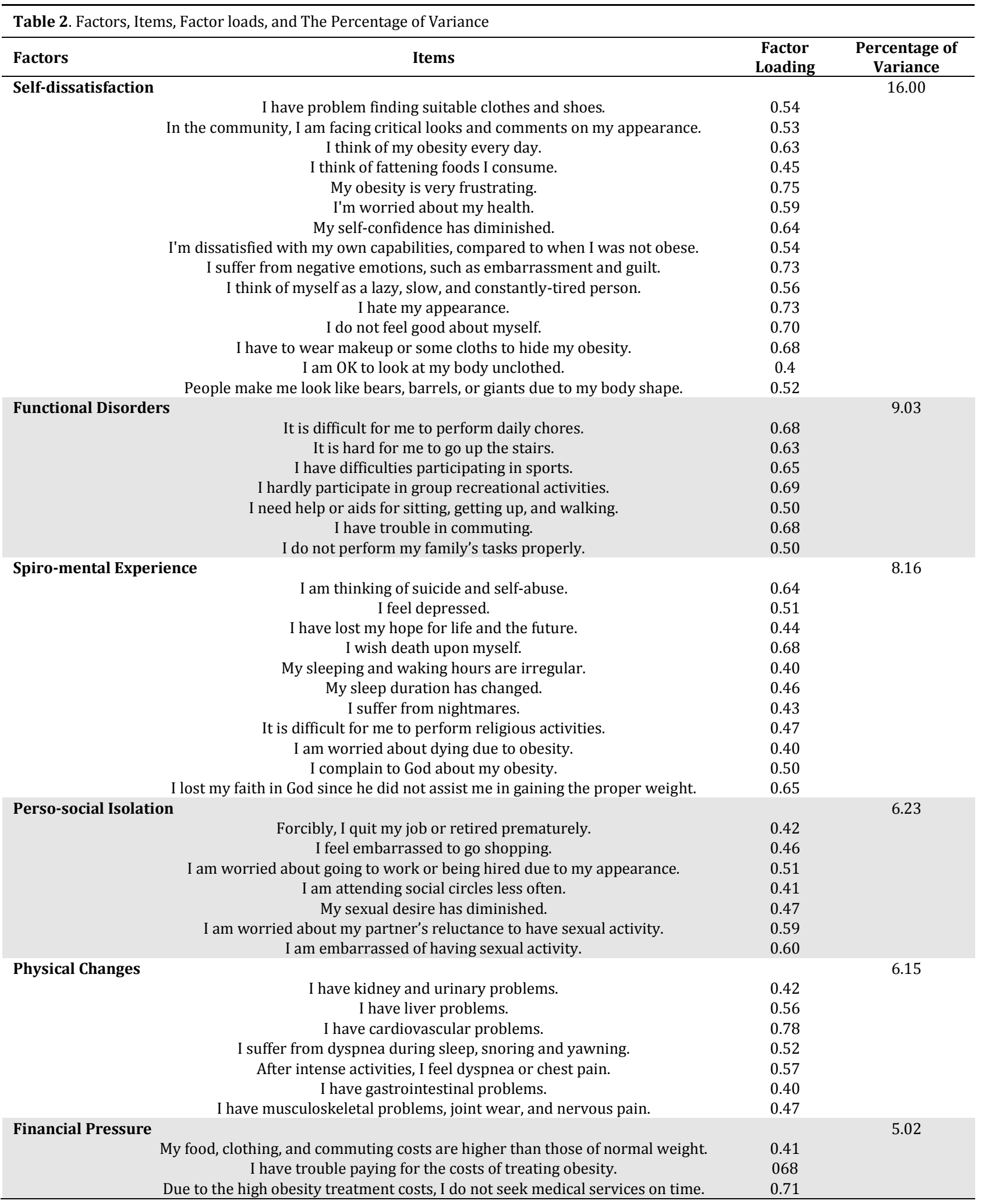

\begin{tabular}{|c|c|c|c|c|c|}
\hline Statistical Index & $\chi^{2}$ & $\chi 2 / \mathrm{df}$ & RMSEA $(90 \% \mathrm{CI})$ & CFI & IFI \\
\hline \multirow[b]{2}{*}{ Acceptable interval } & & Good $<3$ & Good $<0.08$ & & \\
\hline & & & $\begin{array}{l}\text { Moderate } 0.08-0.1 \\
\quad \text { Weak }>0.1\end{array}$ & $>0.9$ & $>0.9$ \\
\hline Goodness & $2414 / 125$ & 2.08 & 0.06 & 0.91 & 0.90 \\
\hline
\end{tabular}

Abbreviations: CFI, comparative fit index; IFI, incremental fit index; RMSEA, root mean square error of approximation; $\chi 2 / \mathrm{df}$, ratio of $\chi 2$ to degrees of freedom. 


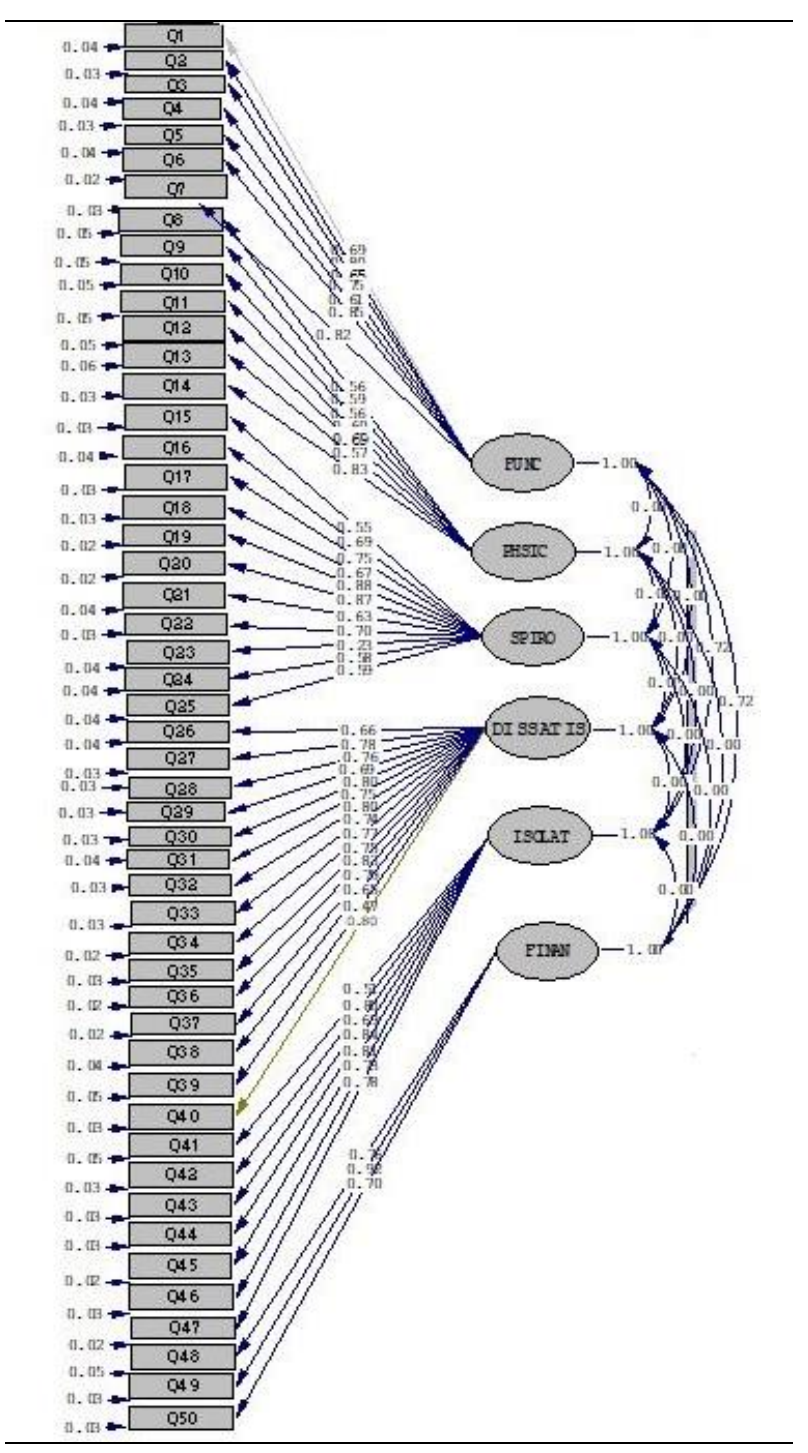

Figure 2. Path diagram of confirmatory factor analysis of the questionnaire
$19.70 \pm 6.22$, spiro-mental experience: $35.47 \pm 8.08$, perso-social isolation: $24.15 \pm 7.49$, physical changes: $23.92 \pm 5.43$, and financial pressure: $8.75 \pm 3.05$. In addition, the total mean value of the questionnaire was obtained at $148.42 \pm 33.10$.

A significant direct correlation was revealed between the scores of MOQOLQ and SF-12 using the Pearson correlation test $(\mathrm{r}=0.71, \mathrm{P}<0.001)$; therefore, the convergent validity was confirmed. Moreover, the correlation coefficient of MOQOLQ with BICI was $0.71(\mathrm{P}<0.001)$. Therefore, the divergent validity of MOQOLQ was approved.

Furthermore, to assess the known-group validity, the results demonstrated the association between QOL scores and BMI categories. Based on the previous assumption, higher mean scores of QOL were found in patients with $\mathrm{BMI}<40(129.41 \pm 62.58)$, compared to those with $\mathrm{BMI} \geq 40 \quad(118.33 \pm 57.12)$ $(\mathrm{r}=-0.13, \mathrm{P}=0.01)$. However, the results did not show any significant correlation between QOL and demographic data, including age, gender, marital status, education level, job, and city $(\mathrm{P}>0.05)$.

\subsubsection{Reliability}

The Cronbach's alpha coefficient of the present questionnaire was calculated at 0.94 , representing an appropriate level of internal consistency. Furthermore, analysis of SEM, SDC, and MIC revealed desirable absolute stability and a sufficiently good agreement of half of the dimensions, as well as the total questionnaire (MIC should be > SDC for positive agreement). The agreement was confirmed using half of six factors and the total score of the questionnaire (Table 4). In addition, the ICC between scores of the test-retest was calculated at 0.97 (Table 5). The data indicated a normal distribution by One-Sample Kolmogorov-Smirnov Test $(\mathrm{P}=0.20)$.

Table 4. Reliability, SEM, SDC, MIC, and Agreement of Six Factors

\begin{tabular}{lccccccc}
\hline Dimensions & Number of Items & Cronbach's Alpha Coefficient & SEM & SDC & MIC & Agreement & P-Value \\
\hline Self-dissatisfaction & 15 & 0.91 & 1.43 & 3.94 & 7.15 & Positive & $<0.001$ \\
Functional disorders & 7 & 0.82 & 1.75 & 4.83 & 3.11 & Negative & $<0.001$ \\
Spiro-mental experiences & 11 & 0.85 & 2.42 & 6.67 & 4.04 & Negative & $<0.001$ \\
Perso-social isolation & 7 & 0.84 & 1.29 & 3.56 & 3.74 & Positive & $<0.001$ \\
Physical changes & 7 & 0.75 & 0.76 & 2.09 & 2.71 & Positive & $<0.001$ \\
Financial pressure & 3 & 0.70 & 1.14 & 3.14 & 1.52 & Negative & $<0.001$ \\
Total & & 0.94 & 5.73 & 15.81 & 16.55 & Positive & $<0.001$ \\
\hline
\end{tabular}

Abbreviations: SEM: Standard of measurement Error, SDC: Smallest detectable change, MIC: Minimal important change

Table 5. Correlation coefficient between the test and retest scores $(n=20)$

\begin{tabular}{lccccccc}
\hline \multirow{2}{*}{ Dimensions } & \multirow{2}{*}{$\begin{array}{c}\text { Number } \\
\text { of Items }\end{array}$} & \multicolumn{2}{c}{ Mest } & Re-test & Std. Error Mean & \multirow{2}{*}{ ICC } & \multirow{2}{*}{ P-Value } \\
\cline { 3 - 6 } & 15 & $36.30 \pm 15.03$ & $36.40 \pm 13.60$ & 3.36 & 3.04 & 0.99 & $<0.001$ \\
\hline Self-dissatisfaction & 7 & $20.70 \pm 7.27$ & $18.70 \pm 5.43$ & 1.57 & 1.21 & 0.92 & $<0.001$ \\
Functional disorders & 11 & $36.65 \pm 9.16$ & $34.30 \pm 7.00$ & 2.04 & 1.56 & 0.91 & $<0.001$ \\
Spiro-mental experiences & 7 & $24.60 \pm 8.06$ & $23.70 \pm 6.93$ & 1.80 & 1.55 & 0.97 & $<0.001$ \\
Perso-social isolation & 7 & $24.40 \pm 5.74$ & $23.45 \pm 5.13$ & 1.28 & 1.14 & 0.97 & $<0.001$ \\
Physical changes & 3 & $8.75 \pm 3.36$ & $8.80 \pm 2.74$ & 0.75 & 0.61 & 0.80 & $<0.001$ \\
Financial pressure & & $151.35 \pm 36.33$ & $145.50 \pm 29.88$ & 8.12 & 6.68 & 0.97 & $<0.001$ \\
\hline Total & & & & & & &
\end{tabular}

Abbreviations: SD: Standard Deviation, ICC: Intra-class correlation coefficient 
4.2.4. Interpretability, Floor and Ceiling Effects, and Scoring

Since MOQOLQ demonstrated a significant difference between the mean score of QOL before and after the surgery $(\mathrm{t}=9.21 ; \mathrm{df}=49 ; \mathrm{P}=0.00)$, the interpretability feature of the questionnaire was confirmed. In addition, none of the participants gained the maximum and minimum scores (i.e., 250 and 50, respectively) at the ceiling and floor effects of MOQOLQ.

Moreover, a linear technique was used to score the questionnaire. The scores 0 and $100(0-$ 33.3=weak, 33.34-66.66=moderate, $66.65-100=$ good) signifies the lowest and the highest ones; therefore, a higher score is indicative of a higher level of QOL. In this regard, $43.9 \%$ and $41.6 \%$ of the participants obtained moderate and weak QOL scores, respectively.

\section{Discussion}

The present study was conducted to design and evaluate the psychometric features of MOQOLQ. The final version of MOQOLQ includes 50 items in six domains, namely self-dissatisfaction, functional disorders, spiro-mental experiences, perso-social isolation, physical changes, and financial pressure.

The validity of MOQOLQ was evaluated by the face, content, and construct validity. Both qualitative and quantitative methods were used to evaluate the face validity. Furthermore, the scale content validity index average (S-CVI/Ave) was used to approve the content validity of this tool (S-CVI=0.98). In this regard, Waltz regarded the score of $\geq 0.90$ as an accepted condition of S-CVI (37). In addition, based on the literature review, the determination of face validity and content validity is the key to validating and using this questionnaire (42).

The first domain with 15 items was the largest one which indicated that MO has the most significant effect on the body image and self-concept of the patients. The highest factor loading $(0.75)$ belonged to the item "My obesity is very frustrating". This result was consistent with those of the impact of weight on quality of life (IWQOL) questionnaire in which the domain of self-confidence/self-esteem consisted of six items. This concordance can signify that morbidly obese patients have negative experiences of their obesity, self-appearance, health status, self-esteem, self-capabilities, and type of clothing. In the same vein, another study revealed that obesity can cause dissatisfaction and unpleasant feelings due to negative body image $(43,44)$. Therefore, health care providers are recommended to support patients and help them to improve their selfesteem.

The second factor of this questionnaire was "functional disorders" with seven items. The factor loading ranged from 0.50 to 0.69 . On the contrary, in the Laval instrument, the third factor was related to "activity and mobility" with 9 items (45). The highest factor loading (0.69) belonged to the item "I hardly participate in group recreational activities". As a result, functional problems of obese patients can cause social isolation which should be prevented by timely prevention and treatment intervention.

The third factor of this questionnaire was "spiromental experiences" with 11 items, and the factor loading ranged from 0.40 to 0.68 . Morbid obese patients are worried about depression, having suicidal thoughts, irregular sleeping patterns, as well as spiritual, health, and mental problems. Although spirituality is a new category of QOL based on the World Health Organization, the majority of the items in this domain have not been included in any of the previous tools as the main subscale (12). In this regard, the measurement of spiritual health can help improve the QOL of obese patients.

The fourth factor consisted of seven items related to perso-social factors and indicated that these patients can experience isolation owing to severe obesity; consequently, it can be concluded that social isolation can negatively influence QOL. In the same way, this domain has been stated in other tools as public distress, social labor, social interaction, social life, and social function $(14,15,44-46)$, and these similarities highlight the importance of this domain. Since these patients face different challenges in society and worry about judgment, health care teams can perform a peculiar role in the prevention and assessment of stigmatization.

The "physical changes" as the fifth factor comprised of seven items and showed that obesity exerts negative effects on all body systems, including urinary, liver, gastrointestinal, cardiovascular, musculoskeletal, and respiratory. In this regard, the majority of items in IWQOL and quality of life, obesity and dietetics (QOLOD) rating scale were related to this domain $(46,47)$; consequently, it seems necessary to prevent and treat physical problems with careful screening and evaluation.

The last factor was "financial pressure" with three items, and factor loading ranged within 0.41- 0.71. This domain has the least number of items, and it has not been considered in the previous instruments. This can be related to the fact that other tools have been designed in developed countries based on experts' viewpoints and not the patients (12). Therefore, the reduction of treatment costs can improve using health services and prevent the incidence of co-morbidities of obesity.

In general, to the best of our knowledge, other MOQOL-related tools have not evaluated all stages of validity and reliability, and most of them have been designed based on the literature review and experts' viewpoints (deductive method). Moreover, they have not included some new domains of QOL, including spiritual and religious factors. Furthermore, there is 
no report regarding the interpretability, as well as ceiling and floor effects. In addition, since the ceiling and floor effects were less than $15 \%$ in this questionnaire, it can be suggested as a suitable instrument for measuring MOQOL. Moreover, the questionnaire can be filled out in about 10 minutes. In this regard, it is noteworthy that a higher responding rate will be achieved if less time is needed to complete the questionnaire.

\subsection{Strengths and Limitations}

Every study has some limitations which must be addressed in the paper. Firstly, the designed questionnaire cannot be generalized to other populations. Secondly, the participants of the two stages were adults; therefore, this tool cannot be suitable for age groups under 18 years old. Therefore, it is suggested that future studies assess the validation features of this question naire in other parts of Iran with larger sample sizes and design a specific questionnaire for morbidly obese adolescents.

Despite these limitations, the present research is one of the few studies that have evaluated all psychometric properties and extracted all dimensions of QOL, including physical, mental, perso/social, economic, and spiritual domains. This instrument was also designed based on the lived experiences of morbidly obese patients in the main referral centers of morbidly obese patients in Iran; therefore, it can be suggested as a feasible and userfriendly questionnaire. Furthermore, we had no missing value in our study since every questionnaire was immediately checked after it was completed by the participants.

\section{Conclusion}

As evidenced by the obtained results, it can be concluded that this valid and reliable questionnaire can be a suitable measurement tool for the assessment of the quality of life among morbidly obese patients in Iran and other countries with similar cultural features. This questionnaire can improve the quality of future clinical research on obesity in various fields of health sciences. Furthermore, health care providers can instruct and offer more efficient services to patients and prevent obesity-related complications.

\section{Acknowledgments}

The present article was extracted from a Ph.D. dissertation submitted by Negar Yazdani. Our deepest appreciation goes to Shiraz and Ahvaz Universities of Medical Sciences and Research Consultation Center (RCC) of Shiraz University of Medical Sciences for invaluable assistance in editing this manuscript. We also extend our gratitude to the participants who took part in this research project.

\section{Footnotes}

Authors' Contribution: Study concept and design: N. Y., F. SH., N. E., A. E., and V. H.; analysis and interpretation of data: N. Y., F. SH., N. E., A. E., and V. H.; drafting of the manuscript: N. Y., and F. SH., critical revision of the manuscript for important intellectual content: N. Y., F. SH., and V. H. statistical analysis: N. Y., F. SH., and A. E.

Conflict of Interests: The authors declare that they have no conflict of interest regarding the publication of the current article.

Funding/Support: The present study was supported in part by Ahvaz Jundishapur University of Medical Sciences (NCRCCD-9416).

\section{References}

1. Tabrizi JS, Sadeghi-Bazargani H, Farahbakhsh M, Nikniaz L, Nikniaz Z. Prevalence and associated factors of overweight or obesity and abdominal obesity in Iranian population: a population-based study of northwestern Iran. Iran J public health. 2018;47(10):1583-92. [PubMed: 30524990].

2. Bananzadeh A, Hoseini SV, Karami MY, Sohrabi Nazari S. Laparoscopic sleeve gastrectomy and gastric cancer incidence. Ann colorectal res. 2015;3(1):e27106.

3. World Health Organization. Obesity and overweight. Geneva: World Health Organization; 2017.

4. Rahmani A, Sayehmiri K, Asadollahi K, Sarokhani D, Islami F, Sarokhani M. Investigation of the prevalence of obesity in Iran: a systematic review and meta-analysis study. Acta Med Iran. 2015;53(10):596-607. [PubMed: 26615371].

5. Jepsen R, Aadland E, Robertson L, Kolotkin RL, Andersen JR, Natvig GK. Physical activity and quality of life in severely obese adults during a two-year lifestyle intervention programme. J Obes. 2015;2015:314194. doi: 10.1155/2015/314194. [PubMed: 25653871].

6. Hruby A, Hu FB. The epidemiology of obesity: a big picture. Pharmacoeconomics. 2015;33(7):673-89. doi: 10.1007/s40 273-014-0243-x. [PubMed: 25471927].

7. Kelley CP, Sbrocco G, Sbrocco T. Behavioral modification for the management of obesity. Prim Care. 2016;43(1):159-75. doi: 10.1016/j.pop.2015.10.004. [PubMed: 26896208].

8. Park CH, Nam SJ, Choi HS, Kim KO, Kim DH, Kim JW, et al. Comparative efficacy of bariatric surgery in the treatment of morbid obesity and diabetes mellitus: a systematic review and network meta-analysis. Obes Surg. 2019;29(7):2180-90. doi: 10.1007/s11695-019-03831-6. [PubMed: 31037599].

9. Kahan S. Overweight and obesity management strategies. Am J Manag Care. 2016;22(7 Suppl):s186-96. [PubMed: 27356116].

10. Lins L, Carvalho FM. SF-36 total score as a single measure of health-related quality of life: Scoping review. SAGE Open Medicine. 2016;4:1-12. doi: 10.1177/2050312116671725. [PubMed: 17757230].

11. Kolotkin RL, Norquist JM, Crosby RD, Suryawanshi S, Teixeira PJ, Heymsfield SB, et al. One-year health-related quality of life outcomes in weight loss trial participants: comparison of three measures. Health Quality Life Outcomes. 2009;7(1):53. doi: 10.1186/1477-7525-7-53. [PubMed: 19505338].

12. Yazdani N, Sharif F, Elahi N, Ebadi A, Hosseini SV. Psychometric properties of quality of life assessment tools in morbid obesity: a review of literature. Evid Based Care. 2018;7(4):7-21. doi: 10.22038/ebcj.2017.26221.1606.

13. Tayyem R, Ali A, Atkinson J, Martin CR. Analysis of healthrelated quality-of-life instruments measuring the impact of bariatric surgery: systematic review of the instruments used 
and their content validity. Patient. 2011;4(2):73-87. doi: 10.2165/11584660-000000000-00000. [PubMed: 21766897].

14. Oria HE, Moorehead MK. Bariatric analysis and reporting outcome system (BAROS). Obesity Surg. 1998;8(5):487-99. doi: 10.1381/096089298765554043. [PubMed: 9819079].

15. Tayyem RM, Atkinson JM, Martin CR. Development and validation of a new bariatric-specific health-related quality of life instrument"bariatric and obesity-specific survey (BOSS)". J Postgrad Med. 2014;60(4):357-61. doi: 10.4103/00223859.143952. [PubMed: 25370541].

16. Haga BM, Furnes B, Dysvik E, Ueland V. Putting life on hold: Lived experiences of people with obesity. Scand J Caring Sci. 2020;34(2):514-23. doi: 10.1111/scs.12756. [PubMed: 31638725].

17. World Health Organization. WHOQOL: measuring quality of life. Geneva: World Health Organization ;2017.

18. Krägeloh CU, Billington DR, Henning MA, Chai PPM. Spiritual quality of life and spiritual coping: evidence for a two-factor structure of the WHOQOL spirituality, religiousness, and personal beliefs module. Health Qual Life Outcomes. 2015;13(1):26. doi: 10.1186/s12955-015-0212-x. [PubMed: 25890224].

19. Jafari-Adli S, Jouyandeh Z, Qorbani M, Soroush A, Larijani B, Hasani-Ranjbar S. Prevalence of obesity and overweight in adults and children in Iran; a systematic review. J Diabetes Metab Disord. 2014;13(1):1-10. doi: 10.1186/s40200-0140121-2. [PubMed: 25610814].

20. Valizadeh Zare N, Mohammadi E, Zarea K, Ehahi N, Manzari Z. The realities of living with a transplanted kidney: A qualitative study. Jundishapur J Chronic Dis Care. 2015;4(3):e28043. doi: 10.5812/jjcdc.28043v2.

21. Speziale HS, Streubert HJ, Carpenter DR. Qualitative research in nursing: Advancing the humanistic imperative: Lippincott Williams \& Wilkins; 2011

22. Graneheim UH, Lundman B. Qualitative content analysis in nursing research: concepts, procedures and measures to achieve trustworthiness. Nurse Educ Today. 2004;24(2):10512. doi: 10.1016/j.nedt.2003.10.001. [PubMed: 14769454].

23. Yazdani N, Sharif F, Elahi N, Hosseini SV, Ebadi A. Exploration of the quality of life in iranian morbid obese people: a qualitative study. Int J Community Based Nurs Midwifery. 2019;7(2):138-49. doi: 10.30476/IJCBNM.2019.44885. [PubMed: 31041324].

24. Connell J, Carlton J, Grundy A, Buck ET, Keetharuth AD, Ricketts $\mathrm{T}$, et al. The importance of content and face validity in instrument development: lessons learnt from service users when developing the Recovering Quality of Life measure (ReQoL). Qual Life Res. 2018;27(7):1893-902. doi: 10.1007/s11136-018-1847-y. [PubMed: 26675691].

25. Taghizadeh Z, Ebadi A, Montazeri A, Shahvari Z, Tavoosi M, Bagherzadeh R. Psychometric properties of health related measures. Part 1: Translation, development, and content and face validity. Payesh. 2017;3:343-57.

26. DeVon HA, Block ME, Moyle-Wright P, Ernst DM, Hayden SJ, Lazzara DJ, et al. A psychometric toolbox for testing validity and reliability. J Nurs Scholarsh. 2007;39(2):155-64. doi : 10.1111/j.1547-5069.2007.00161.x. [PubMed: 17535316].

27. Gilbert GE, Prion S. Making sense of methods and measurement: lawshe's content validity index. Clin Simul Nurs. 2016;12(12):530-1. doi: 10.1016/j.ecns.2016.08.002.

28. Vakili MM, Jahangiri N. Content validity and reliability of the measurement tools in educational, behavioral, and health sciences research. J Med Educ Dev. 2018;10(28):106-19.

29. Munro B. Statistical methods for healthcare research\& application of SPSS in data analysis. and translators 1st ed Tehran: Jamehnegar, Salemi; 2010.

30. Kellar SP, Kelvin EA. Munro's statistical methods for health care research: Wolters Kluwer Health/Lippincott Williams \& Wilkins; 2013.

31. Özdamar K. Paket Programlar ile İstatistiksel Veri Analizi 2 (Çok Değişkenli Analizler. Baskı, Eskişehir; 2010.
32. Ware Jr J, Kosinski M, Keller SD. A 12-item short-form health survey: construction of scales and preliminary tests of reliability and validity. Med care. 1996:34(3):220-33. doi: 10.1097/00005650-199603000-00003. [PubMed: 8628042].

33. Montazeri A, Vahdaninia M, Mousavi SJ, Omidvari S. The Iranian version of 12-item Short Form Health Survey (SF-12): factor structure, internal consistency and construct validity. BMC public health. 2009;9(1):341. doi: 10.1186/1471-2458-9341. [PubMed: 19758427].

34. Littleton HL, Axsom D, Pury CLS. Development of the body image concern inventory. Behav Res Ther. 2005;43(2):229-41. doi: 10.1016/j.brat.2003.12.006. [PubMed: 15629752].

35. Basaknejad S, Ghafari M. The relationship between body image concern and psychological disorders in students. J Behav Sci . 2008;1:179-87.

36. Busutil R, Espallardo O, Torres A, Martínez-Galdeano L, Zozaya $\mathrm{N}$, Á Hidalgo-Vega. The impact of obesity on health-related quality of life in Spain. Health Qual Life Outcomes. 2017;15(1):197. doi: 10.1186/s12955-017-0773-y. [PubMed: 29017494].

37. Waltz CF, Strickland OL, Lenz ER. Measurement in nursing and health research: Springer publishing company; 2010.

38. Koo TK, Li MY. A guideline of selecting and reporting intraclass correlation coefficients for reliability research. J Chiropr Med. 2016;15(2):155-163. doi: 10.1016/j.jcm.2016.02.012. [PubMed: 27330520].

39. Iordens GIT, Den Hartog D, Tuinebreijer WE, Eygendaal D, Schep NW, Verhofstad MH, et al. minimal important change and other measurement properties of the oxford elbow score and the quick disabilities of the arm, shoulder, and hand in patients with a simple elbow dislocation; validation study alongside the multicenter funcsie trial. PLoS One. 2017;12(9): e0182557. doi: 10.1371/journal.pone.0182557. [PubMed: 28886018].

40. Kline RB. Principles and practice of structural equation modeling. fourth ed. New York: Guilford publications; 2015. P:534.

41. Lim CR, Harris K, Dawson J, Beard DJ, Fitzpatrick R, Price AJ. Floor and ceiling effects in the OHS: an analysis of the NHS PROMs data set. BMJ Open. 2015;5(7):e007765. doi:10.1136/bmjopen-2015-007765. [PubMed: 26216152].

42. Polit DF, Beck CT, Owen SV. Is the CVI an acceptable indicator of content validity? Appraisal and recommendations. Res Nurs Health. 2007;30(4):459-67. doi: 10.1002/nur.20199. [PubMed: 17654487].

43. Ahadzadeh AS, Rafik-Galea S, Alavi M, Amini M. Relationship between body mass index, body image, and fear of negative evaluation: Moderating role of self-esteem. Health Psychol Open. 2018;5(1):2055102918774251. doi: 10.1177/205510 2918774251. [PubMed: 29977587].

44. Kolotkin RL, Ervin C, Meincke H, Højbjerre L, Fehnel S. Development of a clinical trials version of the impact of weight on quality of life-lite questionnaire (IWQOL-lite clinical trials version): results from two qualitative studies. Clin Obes. 2017;7(5):290-9. doi: 10.1111/cob.12197. [PubMed: 28544443].

45. Therrien F, Marceau P, Turgeon N, Biron S, Richard D, Lacasse Y. The laval questionnaire: a new instrument to measure quality of life in morbid obesity. Health Qual Life Outcomes. 2011;9(1):1-8. doi: 10.1186/1477-7525-9-66. [PubMed: 21843326].

46. Kolotkin RL, Crosby RD. Psychometric evaluation of the impact of weight on quality of life-lite questionnaire (IWQOL-lite) in a community sample. Qual Life Res. 2002;11(2):157-71. doi: 10.1023/a:1015081805439. [PubMed: 12018739].

47. Ziegler O, Filipecki J, Girod I, Guillemin F. Development and validation of a french obesity-specific quality of life questionnaire: quality of life, obesity and dietetics (QOLOD) rating scale. Diabetes Metab. 2005;31(3):273-83. doi:10.1016/ s1262-3636(07)70194-5. [PubMed: 16142018]. 